Bild und Fall

Ophthalmologe $2021 \cdot 118: 399-403$

https://doi.org/10.1007/s00347-020-01147-w

Online publiziert: 1. Juli 2020

(c) Der/die Autor(en) 2020

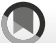

Check tor
updates

Anna Nguyen-Höhl' 1 (D) Mohammed H. Khattab' $\cdot$ Marco Henneke ${ }^{2}$. Nicolas Feltgen ${ }^{1} \cdot$ Hans Hoerauf' $\cdot$ Sebastian Bemme ${ }^{1}$

'Klinik für Augenheilkunde, Universitätsmedizin Göttingen, Göttingen, Deutschland

${ }^{2}$ Klinik für Kinder- und Jugendmedizin, Universitätsmedizin Göttingen, Göttingen, Deutschland

\title{
Einseitige Optikusatrophie bei einem 9-jährigen Patienten
}

noch nicht stattgefunden. Augenverletzungen oder Augenoperationen wurden verneint, bisherige Augenerkrankungen waren nicht bekannt. Die Familienanamnese war unauffällig. Die Mutter berichtete, dass die Familie bis zum 3. Lebensjahr des Kindes in Südamerika lebte. von einer zufälig bemeeten Sehverschlechterung seit ungefähr 2 Wochen. Eine ophthalmologische Untersuchung habe vor Eintreten der Beschwerden

\section{Klinischer Befund}

Der unkorrigierte Visus in der Ferne betrug 1,0 rechts und 0,05 links. Der Nahvisus lag rechts bei 1,0 und links bei 0,1 . Sowohl mit Korrektion als auch mit stenopäischer Lücke war kein besserer Visus zu erheben. Die Augenmotilität war beidseits frei. Es bestanden keine Augenbewegungsschmerzen. Die Pupillomotorik auf dem rechten Auge war unauffällig,

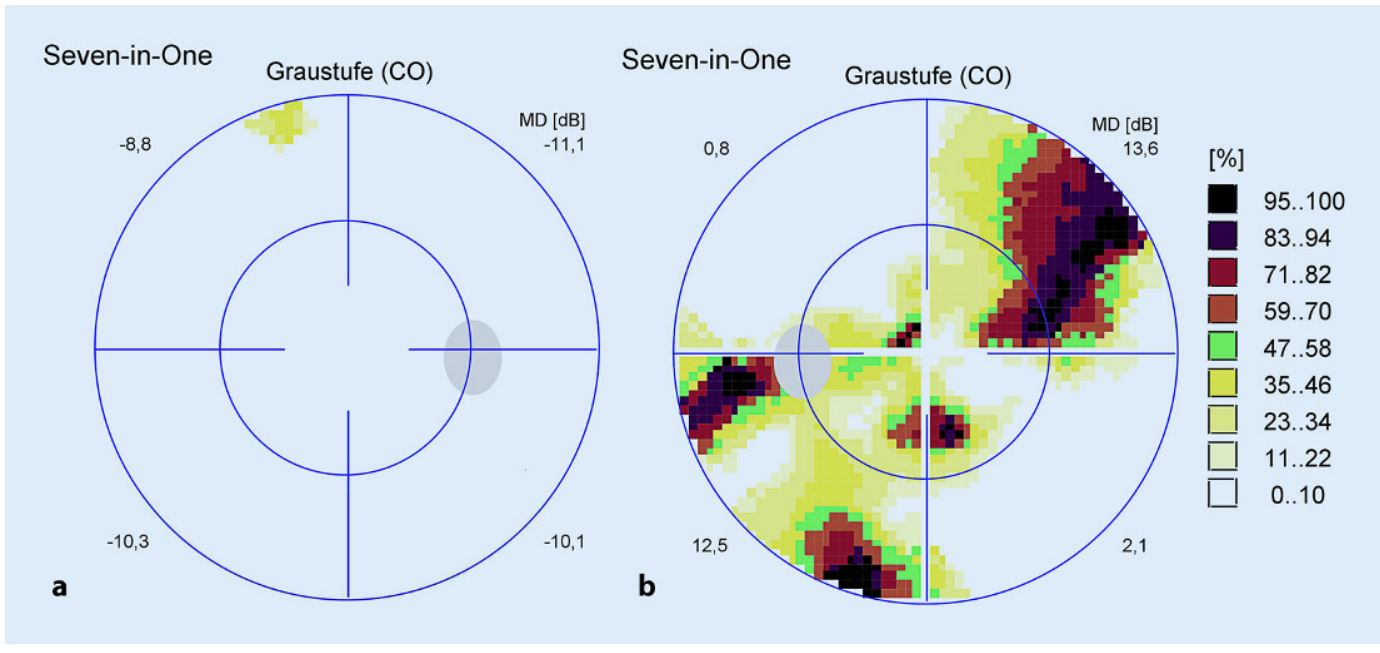

Abb. $1<$ a Das Gesichtsfeld des rechten Auges war unauffällig. b Am linken Auge fanden sich diffuse Ausfälle
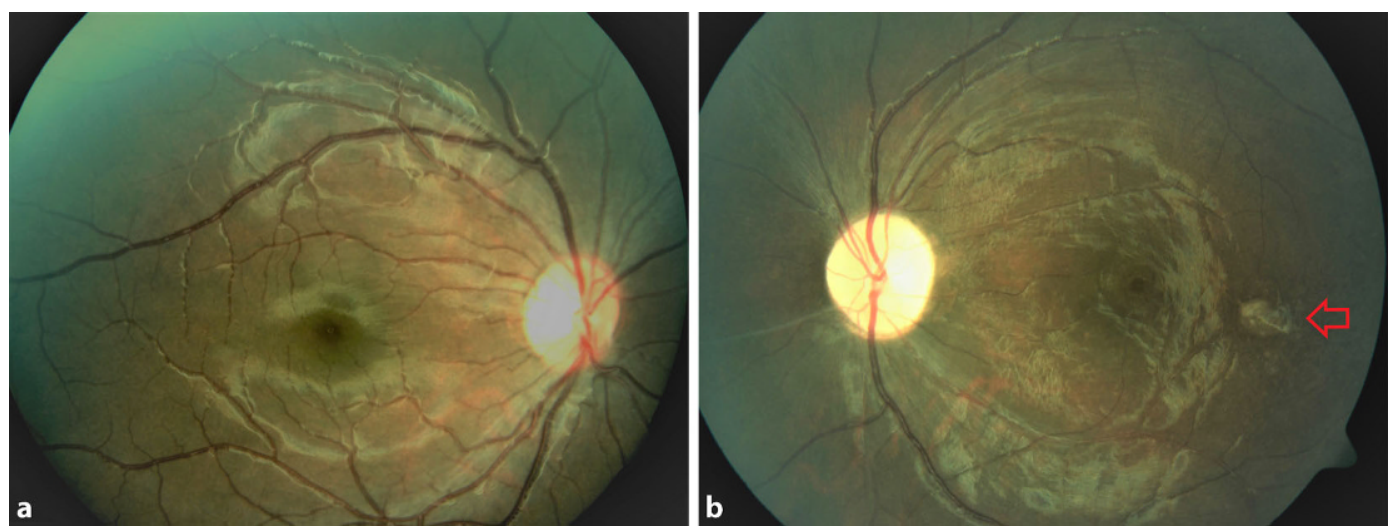

Abb. $2 \triangleleft$ a Die funduskopische Aufnahme des rechten Auges zeigt einen Normalbefund. $b$ Die funduskopische Aufnahme des linken Auges zeigt eine randscharfe, aber blasse Papille bei fehlendem Makula-Wallreflex; in der temporalen Raphe finden sich gelbe Läsionen (roter Pfeil), die retinalen Gefäße erscheinen sehr dünn 


\section{Bild und Fall}
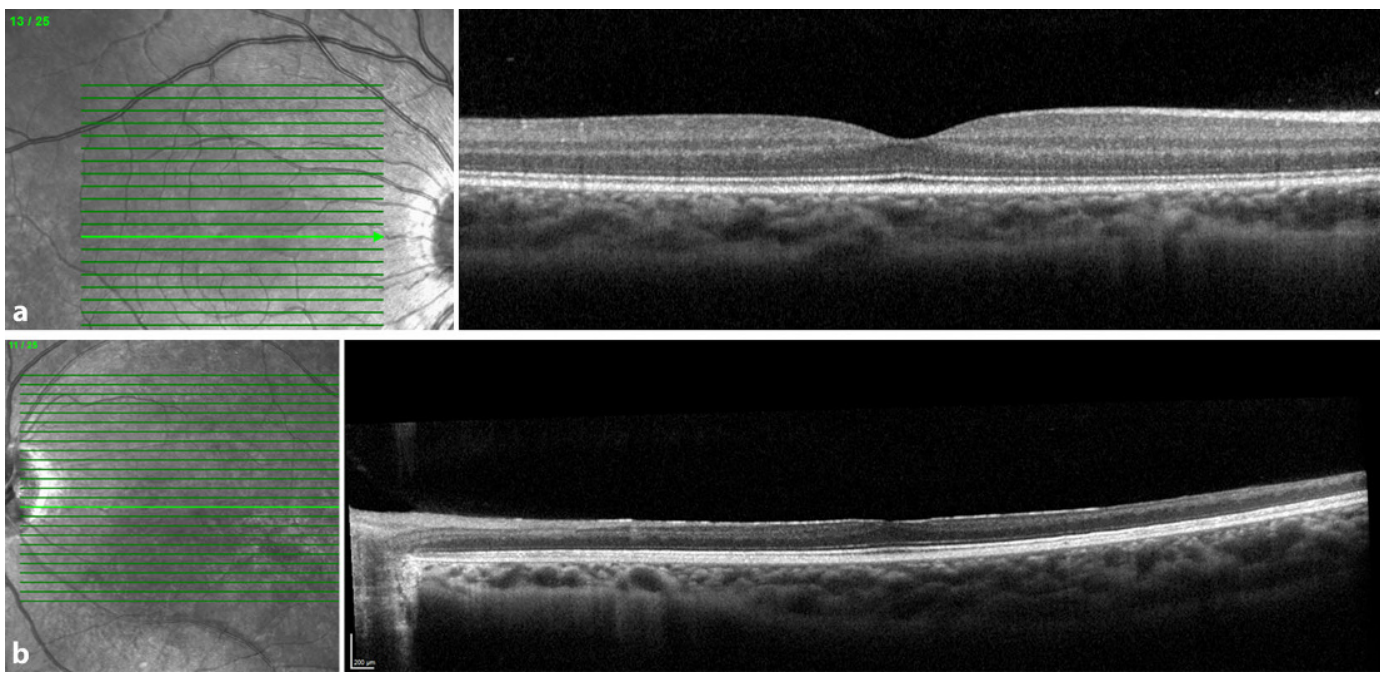

Abb. $3<$ a SD-OCT-Aufnahme der zentralen Netzhaut des rechten Auges mit intakten Netzhautstrukturen. b SD-OCT des linken Auges mit Verdünnung aller Netzhautschichten im Sinne einer Netzhautatrophie und zarter epiretinaler Gliose

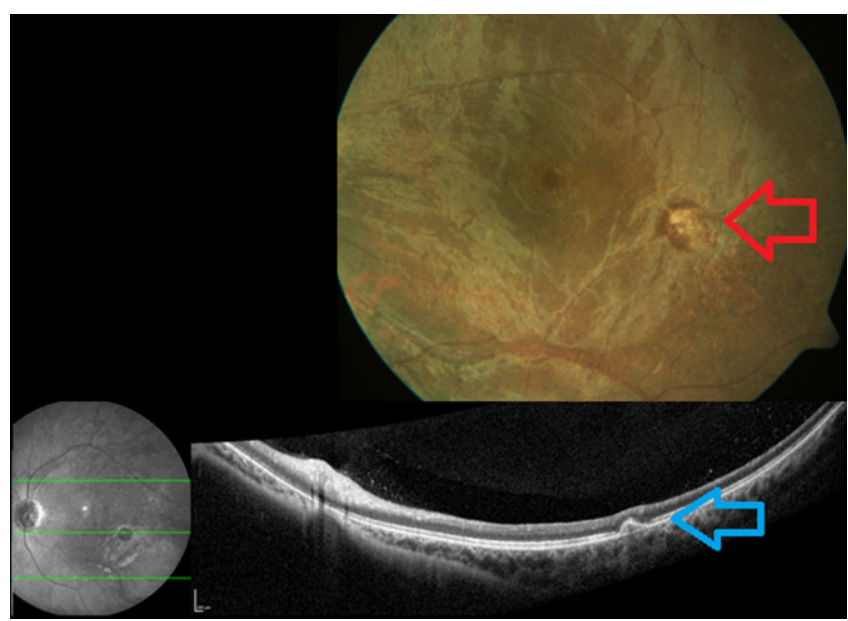

Abb. $4 \triangleleft$ Funduskopische Aufnahme deslinken Auges mit gelblichen Läsionen temporal der Makula (roter Pfeil), die sich in der SD-OCT als hyperreflektive Ablagerungen unter dem RPE darstellen (blauer Pfeil)

auf dem linken Auge war ein relativer afferenter Pupillendefekt (RAPD) nachweisbar. Das $30^{\circ}$-Gesichtsfeld am rechten Auge war frei ( $\mathbf{A} \mathbf{A b b} .1 \mathrm{a})$, am linken Auge fanden wir unspezifische Skotome (- Abb. 1b). Der vordere Augenabschnitt war beidseits unauffällig und reizfrei. Der Glaskörper rechts war unauffällig, links sahen wir eine milde zelluläre Infiltration. Der Augenhintergrund des rechten Auges war unauffällig ( $\bullet$ Abb. 2a). Am linken Auge sahen wir eine randscharfe aber blasse Papille, einen fehlenden Wallreflex der Makula, enggestellte Gefäße und eine fleckige Reflexvermehrung der gesamten Netzhautperipherie (बAbb. 2b). Zudem war temporal der Makula eine gelbliche Läsion zu sehen. Ähnliche Läsionen fanden sich auch vereinzelt in der mittleren Netzhautperipherie. In der Spectral-Domain-optischen Kohärenztomographie (SD-OCT) waren die zentrale und mittelperiphere Netzhaut im Vergleich zum rechten Auge deutlich verdünnt bzw. atrophiert (- Abb. 3a, b). Die gelbliche Läsion temporal der Makula stellte sich in der SDOCT als hyperreflektive Ablagerung unter dem retinalen Pigmentepithel (RPE) dar (•Abb. 4), und der präretinal anliegende Glaskörper war zellig infiltriert.

\section{Diagnostik}

Aufgrund der einseitigen Glaskörperinfiltration wurde eine Uveitisbasisdiagnostik durchgeführt. Die Testung auf Borrelien, Lues, Toxocara, Bartonella henselae und Toxoplasmose waren nicht wegweisend. Auch Rheumafaktor, antinukleäre Antikörper und HLA-B51 waren negativ. Zur weiteren Diagnostik bei einseitiger Optikusatrophie führten wir zudem eine Magnetresonanztomographie des Schä- dels und der Orbita (cMRT) durch, der Befund war unauffällig. Die anamnestische Besonderheit unseres Patienten bestand darin, dass er bis zum 3. Lebensjahr in Südamerika lebte. Wegen des einseitigen subakuten Verlaufs mit diffuser Netzhautbeteiligung bei vorliegender entzündlicher Genese (Vitritis) einerseits und zunächst unauffälligem Uveitislabors andererseits, stellten wir die Verdachtsdiagnose einer diffusen unilateralen subakuten Neuroretinitis (DUSN). Auch die bei unserem Patienten sichtbare Optikusatrophie, Gefäßobliteration, fleckige Reflexvermehrung der Netzhautoberfläche („Oréfice’s sign“) und gelbliche Sub-RPE-Ablagerungen sind typische Befunde bei dieser Erkrankung. Die erweiterte Diagnostik mit Nachweis einer Eosinophilie im Differenzialblutbild und erhöhter IgE-Antikörper ohne bekannte Atopie bzw. Allergien untermauerte schließlich die Diagnose. 


\section{Therapie und Verlauf}

Die DUSN ist eine multifokale Chorioretinitis, die durch Nematoden verursacht wird. Nach Rücksprache mit den Pädiatern unserer Klinik wurde eine Therapie mit Albendazol $400 \mathrm{mg}$ 1-mal täglich p.o. über 4 Wochen eingeleitet, zusätzlich wurde Prednisolon $1 \mathrm{mg} / \mathrm{kg}$ Körpergewicht täglich p.o. verordnet und im Verlauf schrittweise abdosiert. Da bei unserem Patienten ein Fadenwurm funduskopisch nicht sichtbar war, kam eine interventionelle Behandlung mittels fokaler Laserkoagulation (LK) oder eine chirurgische Entfernung des Nematoden mittels Vitrektomie nicht infrage. Bei unserem Patienten lag bereits eine Spätform der Erkrankung mit Optikusatrophie vor, eine Visusbesserung konnte deshalb leider auch nach der Therapie nicht erreicht werden.

\section{Diskussion}

Die diffuse unilaterale subakute Neuroretinitis wurde 1978 erstmalig von Gass beschrieben. Dabei handelt es sich um eine multifokale Chorioretinitis, verursacht durch im subretinalen Raum migrierende Nematoden [1]. Diese gelangen über einen Zwischenwirt wie Hunde oder Waschbären oder auf direktem Wege durch Verschlucken von Nematodeneiern in die Blutwege und letztendlich in das Auge [1]. Unter den Nematoden wurde zuerst Toxocara canis als Ursache für DUSN beschrieben [2]. Zu den ursächlichen Erregern sind neben Baylisascaris procyonis (ein Parasit, der Waschbären und Stinktiere befällt, häufiges Vorkommen im mittleren Westen der USA) und Ancylostoma caninum (ein HundeHakenwurm, häufiges Vorkommen im Südosten der USA) noch einige weitere Nematoden zu zählen [1-4]. Auch in Deutschland sind bereits einige Fälle von DUSN beschrieben, als Erreger vermutet wurde Baylisascaris procyonis [5]. Letztendlich war bei unserem Patienten der Infektions-Zeitpunkt/-Ort nicht nachzuweisen. Sowohl eine frühe Infektion in den ersten Lebensjahren in Südameri- ka, als auch eine spätere Infektion in Deutschland ist denkbar. Die endemischen Orte schließen Lateinamerika und Deutschland ein, jedoch wird von häufigeren Fällen aus Lateinamerika berichtet $[6,7]$. Des Weiteren infizieren sich v.a. Kinder <2 Jahre mit den Nematodeneiern [1]. Die Larven können im Gewebe Monate bis Jahre überleben $[1,2]$. Da die Erkrankungen abwechselnd akute und latente Phasen aufweisen, wird DUSN in vielen Fällen von den Betroffenen nicht bemerkt bis die Spätphase eintritt.

DUSN tritt vorwiegend unilateral auf, seltener wurden bilaterale Verläufe beschrieben [8]. Betroffen sind meist die gesamte Retina sowie das RPE, die retinalen Gefäße, der subretinale Raum, der Sehnerv und die Chorioidea. Vermutet werden eine durch Nematoden und deren Produkte verursachte Inflammation und Degeneration der äußeren Retinaschichten [2]. Die Klassifizierung der DUSN in Früh- und Spätphase erfolgt anhand der klinischen Erscheinung. Die Frühphase ist gekennzeichnet durch einen verminderten Visus, eine moderate Vitritis, $\mathrm{Pa}$ pillitis, retinale Vaskulitis und wiederkehrende gelb-weißliche äußere retinale und chorioidale Läsionen [1]. Die Spätphase dagegen ist durch Optikusatrophie, retinale Arterienverengung, Degenerationen der äußeren Retinaschichten mit diffusen RPE-Unterbrechungen und damit einhergehender erheblicher Visusminderung sowie einem RAPD charakterisiert $[1,2]$. Funduskopisch sind vermehrte Reflexe der internen limitierenden Membran („Oréfice’s sign“) typisch.

Nur die fundoskopische Darstellung eines Fadenwurmes führt zur Diagnosesicherung. Nematoden können in jedem Stadium am Augenhintergrund aufgefunden werden. Der Fadenwurm zeigt sich dabei häufig beweglich. Abhängig von der Spezies beträgt die Fadenwurmlänge 400 bis zu $2000 \mu \mathrm{m}$. Oftmals ist der Fadenwurm jedoch nicht sichtbar, sodass indirekte je nach Früh-oder Spätphase typische Fundusveränderungen eine okuläre Beteiligung der Nematodeninfektion vermuten lassen. Subretinale Spuren (91,7 \%), fokale Veränderungen des RPEs
$(89,3 \%)$, kleine weißliche punktförmige Läsionen $(80,2 \%)$ und eine Optikusatrophie $(76,9 \%)$ sind dabei die häufigsten klinischen Zeichen [9]. In der SDOCT zeigt sich häufig eine Atrophie der Netzhaut am hinteren Pol mit Ausdün-

Zusammenfassung · Abstract

Ophthalmologe $2021 \cdot 118: 399-403$

https://doi.org/10.1007/s00347-020-01147-w @ C Der/die Autor(en) 2020

A. Nguyen-Höhl · M. H. Khattab .

M. Henneke - N. Feltgen $\cdot$ H. Hoerauf . S. Bemme

\section{Einseitige Optikusatrophie bei einem 9 -jährigen Patienten}

\section{Zusammenfassung}

Ein 9-jähriger Patient stellte sich aufgrund einer seit 2 Wochen zufällig bemerkten Sehminderung auf dem linken Auge vor. Funduskopisch waren neben einer leichten Vitritis eine blasse Papille, enggestellte Gefäße und eine Reflexvermehrung der Netzhautoberfläche sichtbar. In der Blutuntersuchung waren die Werte bis auf eine Eosinophilie und einen erhöhten Immunglobublin E(IgE)-Antikörpertiter normal.

\section{Schlüsselwörter}

Chorioretinitis · Vitritis - Optikusatrophie . Visusminderung $\cdot$ Skotom

\section{Unilateral optic atrophy in a 9- year-old patient}

\section{Abstract}

A 9-year-old patient presented with a reduction of visual acuity in the left eye, which was incidentally noticed 2 weeks previously. Funduscopy revealed a mild vitritis, a pale optic disk, narrowed blood vessels and an increased reflex of the retinal surface. The values in the blood examination were normal apart from eosinophilia and an elevated immunoglobulin E ( $\mathrm{lg} E$ ) antibody titer.

\section{Keywords}

Chorioretinitis · Vitritis · Optic atrophy · Loss of visual acuity $\cdot$ Scotoma 
nung der retinalen Nervenfaserschichtdicke und Aufhebung der fovealen Senke. Des Weiteren lassen sich in der SD-OCT gelegentlich subretinale Tunnel ("Garcia's sign“) erkennen, bzw. der Fadenwurm selbst kann als intraretinale Hyperreflektivität dargestellt werden [10]. Eine im Blut nachgewiesene Eosinophilie und IgE-Antikörper-Erhöhung weist auf eine parasitäre Infektion hin.

\section{》) Diagnose: Diffuse unilaterale subakute Neuroretinitis (DUSN)}

Als ergänzende Diagnostik kann eine Elektroretinographie (ERG) durchgeführt werden. Aufgrund des jungen Alters unseres Patienten wurde darauf verzichtet. $\mathrm{Zu}$ erwarten wäre dabei eine reduzierte B-Welle proportional zur retinalen Beteiligung [11].

Differenzialdiagnostisch ist in der Frühphase aufgrund der fokalen Chorioretinitis an Sarkoidose, Toxoplasmose, Histoplasmose, multifokale Chorioiditis, serpinginöse Chorioiditis, akute posteriore multifokale plakoide Pigmentepitheliopathie, Multiple-EvanescentWhite-Dot-Syndrom, unspezifische Optikusneuritis und Papillitis zu denken. Die Spätphase einer DUSN kann einer posttraumatischen Chorioretinopathie, okklusiven Gefäßerkrankung, Chorioretinitis bei Sarkoidose, Retinopathia pigmentosa, LHON oder toxischen Retinopathie [12, 13] ähneln. Bei Befall des zentralen Nervensystems wurden neuropsychiatrische und neurodegenerative Symptome, u. a. Schizophrenie, kognitive Defizite und demenzielle Erkrankungen, beobachtet [14]. Schwere Infektionen beim Kind können sich durch plötzliche Lethargie, Nackensteifigkeit, Torticollis, Opisthotonus, Ataxie, verminderte Kopfkontrolle, Verlust der Feinmotorik, Unfähigkeit alleine zu sitzen, stehen oder gehen äußern [14, 15].

Therapie der Wahl bei Darstellung des Fadenwurmes ist eine Laserkoagulation (LK) des Parasiten. Bei der LK wird der Fadenwurm durch konfluierende Laserherde von $200 \mu \mathrm{m}$ Größe bei $200 \mathrm{~mW}$ und $0,2 \mathrm{~s}$ Dauer mit einem 532- oder 810$\mathrm{nm}$-Laser abgeriegelt, gefolgt von einer lückenlosen weiteren Umstellung. Befin- det sich der Fadenwurm nahe der Fovea mit Gefahr einer bleibenden zentralen Sehminderung nach LK, können sehr zarte Laserherde appliziert werden, um den Fadenwurm Richtung mittlere Peripherie $\mathrm{zu}$ bewegen und anschließend dort die LK des Fadenwurmes durchzuführen [13]. Auch die Vitrektomie wurde als Therapieoption beschrieben [13]. In den meisten Fällen lässt sich aber der Fadenwurm nicht lokalisieren, da Nematoden von Licht wegmigrieren. In diesem Fall beschränkt sich die Behandlung auf eine systemische Therapie mit Antihelminthika, z. B. Albendazol [5]. Begleitend können Kortikosteroide eingesetzt werden, um eine antiinflammatorische Wirkung zu erzielen [16, 17]. Bei Kindern sollte die Therapie unbedingt in enger Abstimmung mit einem Kinderarzt erfolgen.

\section{Fazit für die Praxis}

- DUSN ist eine multifokale Chorioretinitis, verursacht durch Nematoden.

- Typisch ist der einseitige subakute Verlauf mit Beteiligung der gesamten Netzhaut.

- Die Reiseanamnese kann bei der Diagnosestellung wegweisend sein.

- Diagnostisch hilfreich ist der Nachweis einer Eosinophilie und einer lgEErhöhung.

- Frühphase mit moderaten Entzündungszeichen (Vitritis, Papillitis, Vaskulitis).

- Spätphase mit Optikus- und Netzhautatrophie einhergehend mit einem RAPD.

- Therapie der Wahl ist die Laserkoagulation, sofern der Fadenwurm funduskopisch sichtbar ist.

- Alternativ kommen Antihelminthika (z. B. Albendazol) als systemische Therapie zum Einsatz.

\section{Korrespondenzadresse}

\section{Anna Nguyen-Höhl}

Klinik für Augenheilkunde, Universitätsmedizin Göttingen

Göttingen, Deutschland

Anna.Nguyen@med.uni-goettingen.de

Funding. Open Access funding provided by Projekt DEAL.
Einhaltung ethischer Richtlinien

Interessenkonflikt. A. Nguyen-Höhl, M.H. Khattab, M. Henneke, N. Feltgen, H. Hoerauf und S. Bemme geben an, dass kein Interessenkonflikt besteht.

Für diesen Beitrag wurden von den Autoren keine Studien an Menschen oder Tieren durchgeführt. Für die aufgeführten Studien gelten die jeweils dort angegebenen ethischen Richtlinien. Für Bildmaterial oder anderweitige Angaben innerhalb des Manuskripts, über die Patienten zu identifizieren sind, liegt von innen und/oder ihren gesetzlichen Vertretern eine schriftliche Einwilligung vor.

Open Access. Dieser Artikel wird unter der Creative Commons Namensnennung 4.0 International Lizenz veröffentlicht, welche die Nutzung, Vervielfältigung, Bearbeitung, Verbreitung und Wiedergabe in jeglichem Medium und Format erlaubt, sofern Sie den/die ursprünglichen Autor(en) und die Quelle ordnungsgemäß nennen, einen Link zur Creative Commons Lizenz beifügen und angeben, ob Änderungen vorgenommen wurden.

Die in diesem Artikel enthaltenen Bilder und sonstiges Drittmaterial unterliegen ebenfalls der genannten Creative Commons Lizenz, sofern sich aus der Abbildungslegende nichts anderes ergibt. Sofern das betreffende Material nicht unter der genannten Creative Commons Lizenz steht und die betreffende Handlung nicht nach gesetzlichen Vorschriften erlaubt ist, ist für die oben aufgeführten Weiterverwendungen des Materials die Einwilligung des jeweiligen Rechteinhabers einzuholen.

Weitere Details zur Lizenz entnehmen Sie bitte der Lizenzinformation auf http://creativecommons.org/ licenses/by/4.0/deed.de.

\section{Literatur}

1. Gass JDM, Gilbert WR Jr, Guerry RK, Scelfo R (1978) Diffuse unilateral subacute neuroretinitis. Ophthalmology 85(5):521-545

2. Davis JL, Gass JDM (1996) Diffuse unilateral subacute neuroretinitis. In Pepose JS, Holland GN, Wilhelmus KR (Hrsg) Ocular infection and immunity, chapter 88 . Mosby, St. Louis, S 1243124

3. Gass JDM (1987) Stereoscopic atlas of macular diseases. Diagnosis and treatment

4. Goldberg MA, Kazacos KR, Boyce WM, Ai E, Katz B (1993) Diffuse unilateral subacute neuroretinitis: morphometric, serologic, and epidemiologic support for Baylisascaris as a causative agent. Ophthalmology 100(11):1695-1701

5. Küchle M, Knorr HL, Medenblik-Frysch S, Weber A, Bauer C, Naumann GO (1993) Diffuse unilateral subacute neuroretinitis syndrome in a German most likely caused by the raccoon roundworm, Baylisascaris procyonis. Graefes Arch Clin Exp Ophthalmol 231(1):48-51

6. Souza EC, CunhaS, Gass JD (1992) Diffuse unilateral subacute neuroretinitis in south america. Arch Ophthalmol 110(9):1261-1263

7. OueghlaniE, O'Sullivan E, Pavesio CE (2010) Diffuse unilateral subacute neuroretinitis in the United Kingdom. Int Ophthalmol 30(5):615-619

8. Souza EC de (1999) Diffuse bilateral subacute neuroretinitis. Arch Ophthalmol 117(10):1349 
9. de Amorim Garcia Filho CA, Gomes AHB, Garcia ACMDA, de Amorim Garcia CA (2012) Clinical features of 121 patients with diffuse unilateral subacute neuroretinitis. Am J Ophthalmol 153(4):743-749

10. Berbel RF, Casella AMB, de Souza EC, Farah ME (2014) Evaluation of patients with diffuse unilateral subacute neuroretinitis by spectral domain optical coherence tomography with enhanced depth imaging. Clin Ophthalmol 8:1081

11. Roy FH, Fraunfelder FW, Fraunfelder FT (Hrsg) (2007) Roy and Fraunfelder's current ocular therapy. Elsevier B.V., Amsterdam

12. Sabrosa NA, Arevalo JF (2010) DUSN: a potentially blinding parasitic infection. Rev Ophthalmol 17(2):58-62

13. Matsumoto BT, Adelberg DA, Del LP (1995) Transretinal membrane formation in diffuse unilateral subacute neuroretinitis. Retina 15(2):146-149

14. Graeff-Teixeira C, Loureiro Morassutti A, Kazacos KR (2016) Update on baylisascariasis, a highly pathogenic zoonotic infection. Clin Microbiol Rev 29(2):375-399

15. Kazacos KR, Jelicks LA, Tanowitz HB (2013) Baylisascaris larva migrans. In Garcia HH, Tanowitz HB, Del Brutto $\mathrm{OH}$ (Hrsg), Handbook of clinical neurology 114 (3rd series). Neuroparasitology and tropical neurology. Elsevier B.V., Amsterdam, S251-262

16. Vedantham V, Vats MM, Kakade SJ, Ramasamy K (2006) Diffuse unilateral subacute neuroretinitis with unusual findings. Am J Ophthalmol 142(5):880-883

17. Venkatesan P (1998) Albendazole. J Antimicrob Chemother 41(2):145-147

\section{Theo und FriedI Schöller-Preis für Alternsforschung 2021}

Die Theo und Friedl Schöller-Stiftung vergibt in Zusammenarbeit mit dem Zentrum für Altersmedizin im Klinikum Nürnberg im Jahr 2021 den

\section{Theo und Friedl Schöller-Preis \\ Schwerpunkt: Altersmedizin}

Mit dem Theo und Friedl Schöller-Preis werden abgeschlossene wissenschaftliche Forschungsarbeiten aus den letzten beiden Jahren ausgezeichnet, die sich besonders mit den Belangen multimorbider, hochaltriger Menschen befassen und somit neue Aspekte in Forschung und Lehre eröffnen. Entscheidend ist der Innovationswert, der potentielle Benefit für Patienten und Personal sowie die wissenschaftlich-methodische Qualität. Eine Publikation ist nicht erforderlich.

\section{Preisgeld: $€$ 20.000,-}

Ausschreibungsende: 16.06 .2021

Bewerben können sich im deutschsprachigen Raum tätige (angehende) Wissenschaftler aus den Bereichen Medizin, Lebens-, Natur- und Ingenieurwissenschaften sowie Geistes-, Pflege-, Sozialwissenschaften und der Gesundheitsökonomie. Die Bewerbung um den Theo und Friedl Schöller-Preis ist nur durch die Forscherin oder den Forscher selbst möglich. Es handelt sich um einen personenbezogenen Preis. Arbeiten von Gruppen können ebenfalls eingereicht werden, in diesem Fall muss die Einwilligung aller Beteiligten vorliegen.

Alle Vorschläge sind mit einem eigenständigen Antrag, einer Darstellung der wissenschaftlichen Arbeit bzw. der Publikation, der Kurzbiographie des Bewerbers sowie einer Empfehlung einzureichen. Jeder Bewerber oder jede Arbeitsgruppe darf im Rahmen der Ausschreibung nur eine Arbeit oder ein Forschungsprojekt vorlegen. Einzureichen ist ferner eine Versicherung, dass die Arbeit oder das Projekt nicht für eine andere Ausschreibung eingereicht oder vorgesehen ist oder von anderer Seite bereits ausgezeichnet wurde.

Die Arbeit soll zumindest in der Zusammenfassung in deutscher Sprache vorliegen. Der für die Bewerbung notwendige Leitfaden ist auf der unten genannten Website abzurufen.

Form der Bewerbung:

- max. 10 Seiten Umfang (Darstellung der wissenschaftlichen Arbeit)

DIN A4, Schriftgröße 12 Pt., einzeiliger Zeilenabstand

.pdf-Format, alle Unterlagen in einem Dokument (max. 15 MB)

- Die Bewerbung ist in elektronischer Form unter folgender Adresse einzureichen:

Zentrum für Altersmedizin im Klinikum Nürnberg

z.Hd. Frau Elke Kaufmann

Prof.-Ernst-Nathan-Straße 1

90419 Nürnberg

Tel: 0911/398-3917

E-Mail: alterszentrum@klinikum-nuernberg.de

www.altersmedizin-nuernberg.de

Das Zentrum für Altersmedizin steht für Rückfragen gerne zur Verfügung. 